\title{
An Improved Method of Adhesion Force Measurement by Atomic Force Microscopy (AFM)
}

\author{
Hong Jin, ${ }^{\text {a,b,c }}$ Xiongyao Li, ${ }^{\text {a,b,* }}$ Yuanyun Wen, ${ }^{\text {a,b }}$ Hong Tang, ${ }^{\text {a,b }}$ and Xiaojia Zenga,b \\ ${ }^{a}$ Center for Lunar and Planetary Sciences, Institute of Geochemistry, Chinese Academy of Sciences, Guiyang 550081, P.R. China \\ ${ }^{\mathrm{b}}$ Center for Excellence in Comparative Planetology, Chinese Academy of Sciences, P.R. China \\ ${ }^{c}$ University of Chinese Academy of Sciences, Beijing 100049, P.R. China
}

Received: November 30, 2021; Revised: February 09, 2022; Accepted: February 09, 2022; Available online: February 10, 2022.

DOI: 10.46770/AS.2022.011

ABSTRACT: The adhesion properties of lunar dust grains are a key to determine its motion state. Atomic force microscopy (AFM) is conducted to evaluate the adhesion properties of such ultrasmall grains. However, effective method to measure the adhesion properties of lunar grains has not yet been developed because of the difficulties in eliminating the effect of adsorption of water and gases on the grain surface. In this study, an improved method was proposed to measure the adhesion force of grains while effectively eliminating the gas molecule adsorption effect. In the proposed method, using a focused ion beam, a small grain was mounted onto the tip of an AFM probe and used to measure the adhesion force of the grain. To determine the effects of environmental pressure and temperature, the adhesion force between a silica ball and a silica wafer was measured under different conditions. Based on the results, the gas molecule adsorption effect can be effectively eliminated during adhesion force measurement through $\mathrm{AFM}$ at a temperature of $200^{\circ} \mathrm{C}$ and an environmental pressure of $<2.4 \times 10^{-4} \mathrm{~Pa}$, at which strong adhesion of the grain is achieved. The proposed method is suitable for the measurement of adhesion force in lunar grain samples.

\section{INTRODUCTION}

Lunar dust adhesion causes damage to spacecrafts and astronauts. ${ }^{1}$ The analysis of this adhesion property facilitates the understanding of the migration of lunar dust and determination of the cause of the lunar horizon glow (LHG) phenomenon. ${ }^{2}$ During the Apollo mission, the astronauts who landed on the Moon found that the damage caused by lunar dust was far beyond their expectations. ${ }^{3}$ The strong adhesion of lunar dust may cause thermal control system malfunction, failure of the sealing structure, mechanism jamming, material wear, and tip discharge. ${ }^{4}$ In addition, the cause of the LHG phenomenon detected during Surveyor and Apollo missions is still debated by scientists. The common opinion is that LHG is caused by the solar light scattering of dense lunar dust clouds suspended at an altitude of about $100 \mathrm{~km}$ above the lunar surface. ${ }^{5,6,7}$ However, the $100 \mathrm{~km}$ static-electronic migration height of lunar dust is contradicted by, especially, the result of Lunar Dust EXperiment, which shows no evidence of a large amount of charged dust $3 \mathrm{~km}$ above the lunar orbit. ${ }^{8}$ Theoretically, the migration height and density of lunar dust is controlled by adhesion. $^{2}$ On the lunar surface, lunar dust migration must overcome the gravitational force induced by the grain itself, the Coulomb force, and adhesion among the grains. For $10-\mu \mathrm{m}$ lunar dust grains, adhesion is considerably strong to overcome the gravitational and Coulomb forces. ${ }^{2}$ The gravitational force is only approximately $10^{-6} \mu \mathrm{N}$ and the Coulomb force between two adjacent grains with $10^{5}$ electrons is estimated to be $0.023 \mu \mathrm{N}$. 9 Based on Walton's study, the adhesion force between two adjacent lunar dust grains is theoretically estimated to be $12-120 \mu \mathrm{N}$ when the lunar dust surface energy is $20-200 \mathrm{~mJ} / \mathrm{m}^{2} .{ }^{10}$

However, the theoretical value has not yet been verified by experimental analysis because of the limitations of the conventional adhesion force measurement method. The drastic effect of surface adsorbates limits the application of atomic force microscopy (AFM) to measure lunar dust grain adhesion force. Based on the BET adsorption theory, various gas molecules are adsorbed and form a film on the lunar dust grain surface during 
study; it involved heating in a high vacuum atmosphere. This method can help us understand the adhesion properties of grains in the lunar environment.

Fig. 1 Mechanisms of adsorption of water and gases on agglutinitic glass and silica. The oxygen atoms in the silica molecule form bonds with the hydrogen atoms in the water molecules, resulting in adsorption. In addition, other gases can adsorb to defective silicon atoms.

adhesion force measurement by AFM, which is conducted under a high vacuum (Fig. 1). ${ }^{11,12}$ The adsorption of gas molecules may result in a significant reduction in adhesion force, because adsorption is an exothermic process and would cause a loss of energy from the surface. ${ }^{10}$ Therefore, it is necessary to eliminate the effect of surface adsorbates during the AFM-based measurement of adhesion force of lunar dust grain.

Currently, there is no effective method to eliminate the effect of surface adsorbates during the AFM-based measurement of lunar dust grain adhesion force. In previous studies, a high-purity gas protection method was adopted to eliminate the effect of water adsorption. Most researchers have measured adhesion force by limiting the water content of the adsorbent by using a high-purity gas during AFM..$^{13,14,15}$ In such studies, a high-purity nitrogen protection environment weakened the adsorption of water molecules. However, it is difficult to eliminate the adsorption of the other gas molecules. With the improvement in the efficiency of the AFM technique, high-vacuum AFM was developed. Because the adsorption of gas molecules can be reduced in a high vacuum, high-vacuum AFM is mainly conducted for the accurate measurement of interfacial properties, including microtopography, electromagnetism, and tribology. ${ }^{16}$ However, only a few studies on adhesion force measurement by high-vacuum AFM have been conducted because the elimination of the effect of the adsorbed gases is difficult. The adsorption of gas molecules is closely related to their deposition rate, which depends on the environmental pressure. According to previous studies, the deposition rate decreases with pressure, and is still larger than one molecular layer per second at a pressure of $3.0 \times 10^{-4} \mathrm{~Pa}^{17} \mathrm{It}$ indicates that one gas molecular layer is deposited on the grain surface when the grain is exposed into this pressure for more than $1 \mathrm{~s}$. To generate adsorption energy, desorption must be allowed for the absorption of external energy to overcome the heat of adsorption. Therefore, the adsorption of gas molecules cannot be prevented by simply increasing the vacuum. ${ }^{11}$

To prevent the adsorption of gas molecules, an improved AFM method for adhesion force measurements was proposed in this

\section{EXPERIMENTAL}

Basic principle. AFM analyzes the interaction between a probe tip and materials at the micro/nanoscale. When the tip touches the sample surface, adhesion force is generated between the tip and the sample surface. To separate the tip from the sample surface, a force must be applied to overcome the adhesion force. Before the tip separates from the sample surface, the external force is lower than the adhesion force and causes the elastic deformation of the cantilever of the probe. ${ }^{18}$ Such deformation can be reflected by the direction of the laser beam, and the maximum deformation is recorded as soon as the tip separates from the sample surface. Using the known coefficient of elastic deformation of the cantilever, the maximum elastic force of the tip that corresponds to the adhesion force between the tip and the sample surface can be determined according to the maximum deformation of the cantilever. Based on this principle, the adhesion force between a grain and a material surface can be measured by lifting the tip with the grain attached from the sample surface.

Probe preparation. The key process in adhesion force measurement is the mounting of a grain on the tip of the probe. ${ }^{19}$ An ultraviolet (UV)-curable adhesive is usually used for this mounting process (Fig. 2A). However, the mounted grain can easily be contaminated by the adhesive, which is very difficult to remove. ${ }^{20}$ In addition, the volatilization of organic matter may affect the measurement in a vacuum environment. To prevent grain contamination, a mounting method involving focused ion beam scanning electron microscopy (FIB-SEM) was proposed. Before mounting the grain on the tip of the probe, the sample grain was cleaned and placed on a sample holder. Using the FEI Scios FIB-SEM system at the Center for Lunar and Planetary Sciences, Institute of Geochemistry, Chinese Academy of Sciences, the electron beam was adjusted to $20 \mathrm{keV}$ and $0.4 \mathrm{nA}$, at which the grain surface morphology was imaged without spraying a conductive layer. A 30-keV and 30-pA focused ion beam was used to mount the grain on the tip of the probe through Pt deposition. In this study, standard silica pellets (Bangs Co. Fisher, IN) were mounted on the tip of the probe (Fig. 2B and C). The FIB-SEMbased method ensured that the top of the silica pellets was not contaminated and that the probe was not damaged during the mounting process.

Probe calibration. Probe calibration was performed to determine the spring constant of the cantilever using the thermal tune method. ${ }^{21}$ According to the Lorentz and simple harmonic oscillator models, the elastic coefficient of the cantilever is 


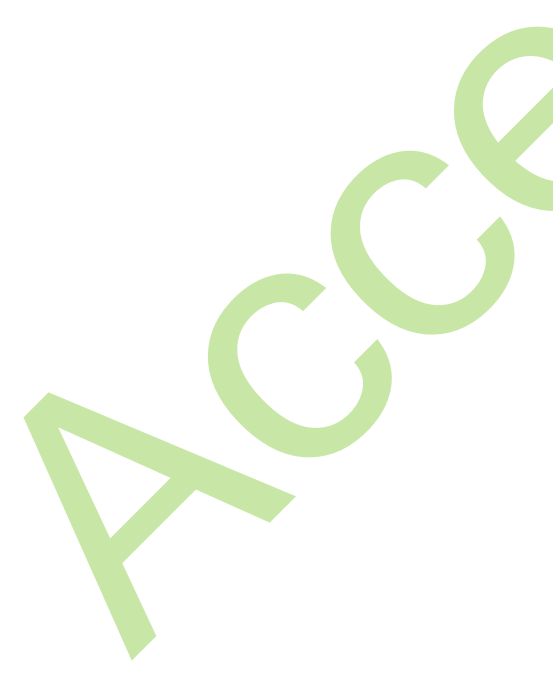

calculated by a computer program. ${ }^{23}$ The resonance frequency of the cantilever for the Oxford AC200 AFM probe (modified with a silica grain) was $151 \mathrm{kHz}$, and the elastic modulus was $12.9 \mathrm{~N} / \mathrm{m}$.

Sample measurement. Once the probe was calibrated, it was used to measure the adhesion force between the grain mounted on the tip and the sample through high-vacuum AFM. To measure the adhesion force, AFM was set to the contact mode. After the system automatically inserted the probe, the system pressed the probe against the sample and pulled it out according to the set parameters such as pressing depth and lifting height. The adhesion force between the probe and the sample was then measured.

An environmentally controllable AFM system made by Seiko NanoNavi was used to measure the adhesion force between the modified tip of the probe and a silica wafer (the nominal surface roughness was $<0.5 \mathrm{~nm}$ ). The aim was to weaken the effect of the adsorbed substances on the surface of the grains during the measurement. The holding time after each temperature change was $5 \mathrm{~min}$ to ensure that the system had reached an equilibrium state; laser sensitivity correction was performed after each temperature change. Ten different positions on the substrate were selected to measure the adhesion force. The distance between each of the two points was larger than twice the grain radius to prevent potential inelastic deformation of the substrate.

\section{RESULTS AND DISCUSSION}

To determine the effect of temperature on adhesion, the adhesion force between a standard silica ball and a silica wafer substrate was measured at different temperatures and at an environmental pressure of $<3.2 \times 10^{-4} \mathrm{~Pa}$.

In the experiment, the sample was heated to a maximum temperature of $200^{\circ} \mathrm{C}$ in the high-vacuum AFM system, and then cooled to $60^{\circ} \mathrm{C}$ for three cycles. The adhesion force was measured during the temperature increase process at $60,90,120,150$ and $200{ }^{\circ} \mathrm{C}$ (Table 1). In the temperature range of $60-200{ }^{\circ} \mathrm{C}$, grain deformation can be neglected because the change rate of the elastic modulus of silica was $<1.01 .^{24}$ In addition, the direct effect of temperature on adhesion force was weak in this temperature range. When the temperature coefficient of silica adhesion was $-0.01 \%$ per degree Celsius, the direct effect of temperature on adhesion force was less than $-1.4 \%{ }^{25}$

On the other hand, adhesion changed as the temperature rose because of the desorption of adsorbates on the grain surface..$^{26,27}$ During the heating of the sample, the gas that had adsorbed onto the surface of the sample gradually desorbed, re-establishing the equilibrium according to the new temperature and pressure.

In Fig. 3, the three heating cycles show similar trendsadhesion force increases with an increase in temperature. When 
Table 1 Adhesion forces of silica grains at different temperatures and pressures

\begin{tabular}{lcccccc}
\hline \multirow{2}{*}{ Adhesion nN } & & \multicolumn{5}{c}{ Temperature } \\
\cline { 3 - 7 } & & $60^{\circ} \mathrm{C}$ & $90^{\circ} \mathrm{C}$ & $120^{\circ} \mathrm{C}$ & $150^{\circ} \mathrm{C}$ & $200^{\circ} \mathrm{C}$ \\
\hline \multirow{3}{*}{ Pressure (Pa) } & $3.1 \times 10-4 \mathrm{~Pa}$ & $474.82 \pm 13.21$ & $494.82 \pm 15.26$ & $750.93 \pm 26.84$ & $833.24 \pm 29.16$ & $787.91 \pm 56.66$ \\
& $2.4 \times 10-4 \mathrm{~Pa}$ & $691.62 \pm 33.84$ & $896.73 \pm 56.48$ & $1252.49 \pm 42.06$ & $1467.08 \pm 38.35$ & $1515.44 \pm 58.54$ \\
& $2.0 \times 10-4 \mathrm{~Pa}$ & $923.27 \pm 25.72$ & $1050.82 \pm 27.44$ & $1365.25 \pm 47.17$ & $1547.75 \pm 30.13$ & $1516.55 \pm 54.05$ \\
\hline
\end{tabular}

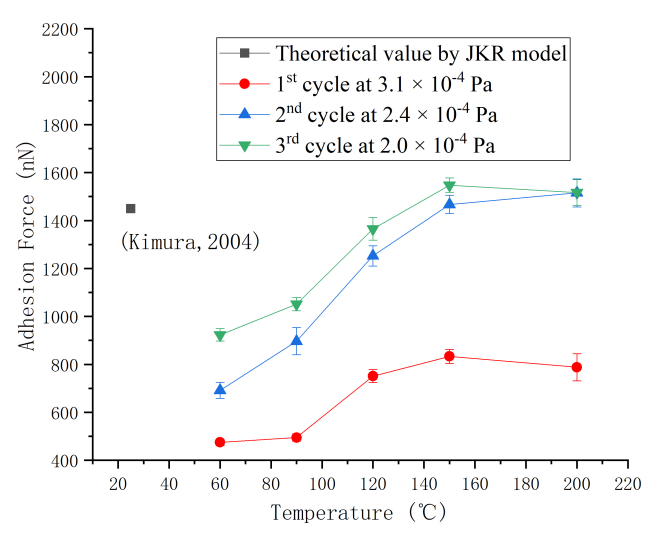

Fig. 3 Relationship between adhesion force and temperature.

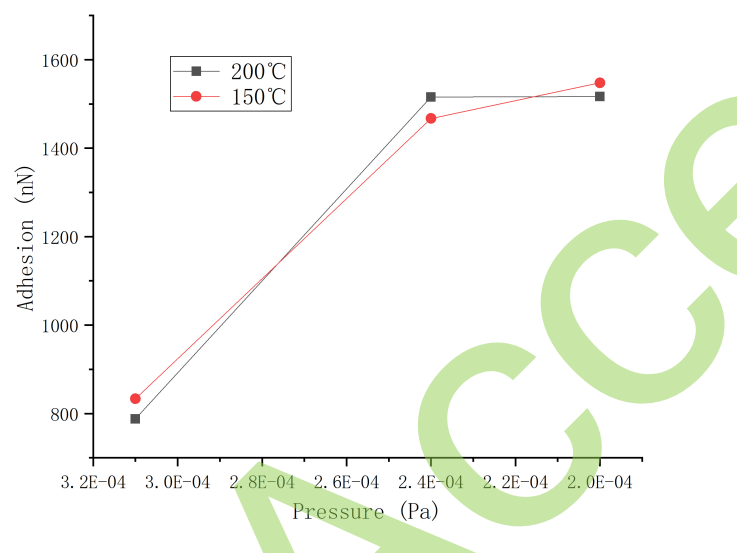

Fig. 4 Effect of pressure on adhesion force measured at 150 and $200^{\circ} \mathrm{C}$.

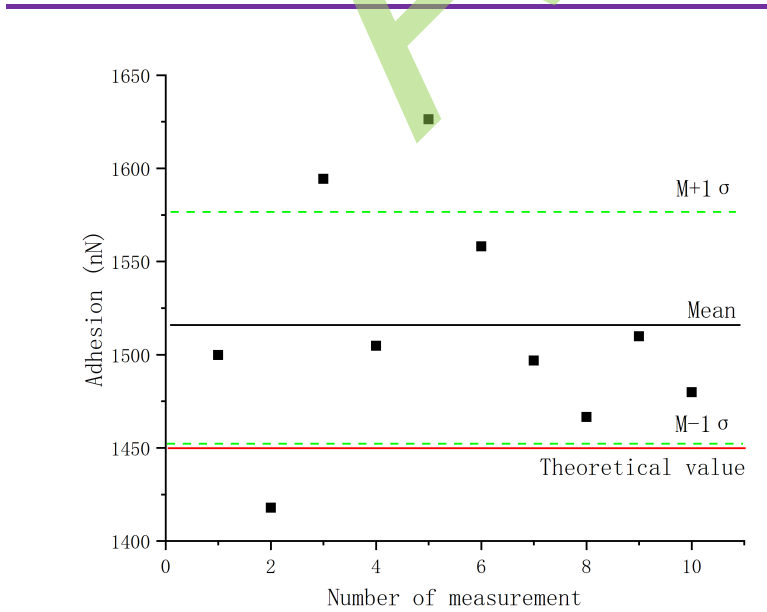

Fig. 5 Analysis of adhesion force measurement results at $200{ }^{\circ} \mathrm{C}$ and $2.4 \times 10^{-4} \mathrm{~Pa}$. the temperature was increased from 60 to $150{ }^{\circ} \mathrm{C}$ (first stage), the adhesion force rapidly increased; a stable trend was observed when the temperature increased from 150 to $200^{\circ} \mathrm{C}$ (second stage). In the first stage, the gas molecules escaped from the grain surface by absorbing enough energy to overcome the heat of adsorption when the temperature was increased. The higher the heating temperature, the higher the kinetic energy of the gas molecules and the easier the desorption of the gas molecules. As a result, the content of adsorbed gas molecules decreased with the increase in temperature. This would lead to increasing adhesion force because the surface energy of the gas is lower than that of the grain.

In the second stage, the content of adsorbed gas molecules slightly changed at the same pressure because the adsorption and thermal desorption processes had reached equilibrium. In this case, the adsorbed gas content depended on the adsorption rate, which was controlled by pressure. Theoretically, a molecular gas layer on the grain surface can be formed easily in a short time because of the high adsorption rate at high pressure $\left(>3.0 \times 10^{-4} \mathrm{~Pa}\right)$. The layer of adsorbed gas molecules would drastically affect the adhesion of the grain. It was verified in our study that the mean value of adhesion force measured at $3.1 \times 10^{-4} \mathrm{~Pa}$ was $787.91-833.24 \mathrm{nN}$, which is much lower than the theoretical value of $1449.74 \mathrm{nN}^{28}$ When the pressure is lower than $3.0 \times 10^{-4} \mathrm{~Pa}$, adsorption of gas molecules can be effectively prevented because the adsorption rate is sufficiently low at low pressure, and the adsorbed gas molecules can absorb enough energy to escape the grain surface over the temperature range of $150-200{ }^{\circ} \mathrm{C}$. Therefore, the adsorbed gas molecules have a negligible effect on adhesion in such cases. It was verified in our study that the theoretical value is consistent with our experimental results. The mean values of adhesion force measured at $2.0 \times 10^{-4}$ and $2.4 \times 10^{-4} \mathrm{~Pa}$ were $1532.15 \mathrm{nN}$ and $1491.26 \mathrm{nN}$, respectively.

As shown in Fig. 4, the difference in adhesion force measured at $2.4 \times 10^{-4}$ and $2.0 \times 10^{-4} \mathrm{~Pa}$ at $200^{\circ} \mathrm{C}$ is smaller than that measured at $150{ }^{\circ} \mathrm{C}$. At $200^{\circ} \mathrm{C}$, the adhesion force values were 1515.44 and $1516.55 \mathrm{nN}$ at $2.4 \times 10^{-4}$ and $2.0 \times 10^{-4} \mathrm{~Pa}$, respectively. The difference was only $1.11 \mathrm{nN}$. The adhesion force values were 1467.08 and $1547.75 \mathrm{nN}$ at $2.4 \times 10^{-4}$ and $2.0 \times 10^{-4} \mathrm{~Pa}$, respectively, at $150{ }^{\circ} \mathrm{C}$. The difference was $80.37 \mathrm{nN}$, which is larger than that at $200^{\circ} \mathrm{C}$. This indicates that desorption was more effective at $200{ }^{\circ} \mathrm{C}$. The measured value of adhesion force was closer to the theoretical value at this temperature. 
According to the above-mentioned analysis, the effect of gas molecule adsorption can be eliminated effectively at $200{ }^{\circ} \mathrm{C}$ and $2.4 \times 10^{-4} \mathrm{~Pa}$. By using the improved method, the adhesion force between silica grains and the silica wafer was measured. Figure 5 shows good discreteness, and the standard error is $<61.7 \mathrm{nN}$. Most of the data are located in the $1 \mathrm{e}$ confidence interval and the mean adhesion of grain is $1502.27 \pm 28.84 \mathrm{nN}$ within one standard deviation, which is close to the theoretical value of $1449.97 \mathrm{nN}$.

\section{CONCLUSIONS}

In this study, the effect of gas molecule adsorption on adhesion force was found to be strong when pressure was higher than $3.0 \times$ $10^{-4} \mathrm{~Pa}$. For adsorption of heat, the adsorbed gas molecules could not escape from the grain surface even when the pressure was lower than $3.0 \times 10^{-4} \mathrm{~Pa}$. This is because they did not have sufficient energy to overcome the adsorption of heat. The adsorbed gas molecules can be effectively eliminated only by heating under a high vacuum. The experimental results indicated that gas adsorption and desorption reached an equilibrium, and the effect of gas molecule adsorption was eliminated at $200{ }^{\circ} \mathrm{C}$ and $2.4 \times$ $10^{-4} \mathrm{~Pa}$. Under these conditions, the adhesion force between a silica grain having a size of $3.62 \mu \mathrm{m}$ and a silica wafer substrate was measured. The results showed a small standard error, and the mean value was consistent with the theoretical estimate. This improved method is suitable for the measurement of lunar dust grain adhesion force and may help us to further understand the lunar dust environment.

\section{AUTHOR INFORMATION}

Xiongyao $\mathbf{L i}$ is a research professor of planetary science at the Institute of Geochemistry, Chinese Academy of Sciences (IGCAS) in Guiyang, China. He is the director of the Center for Lunar and Planetary Sciences, IGCAS. He completed his $\mathrm{Ph} . \mathrm{D}$. in cosmochemistry from the University of Chinese Academy of Sciences in 2006. His research focused on lunar surface environment, lunar soil properties and space weathering. $\mathrm{He}$ published over 100 papers in SCI journals.

\section{Corresponding Author}

*X.Y. Li

Email address: lixiongyao@mails.gyig.ac.cn

Notes

The authors declare no competing financial interest.

\section{ACKNOWLEDGMENTS}

The authors acknowledge funding support from the Strategic Priority Research Program of the Chinese Academy of Sciences (XDB 41000000), the Natural Science Foundation of China (41931077), the Technical Advanced Research Project of Civil Space (D020201), the Key Research Program of Frontier Sciences, CAS (ZDBS-SSW-JSC007-10 and QYZDY-SSW-DQC028) and Guangxi Scientific Base and Talent Special Projects (AD1850007).

\section{REFERENCES}

1. B. J. O'Brien, Planet. Space Sci., 2018, 156, 47-56. https://doi.org/10.1016/j.pss.2018.02.006

2. T. J. Stubbs, R. R. Vondrak, and W. M. Farrell, Adv. Space Res., 2006, 37, 59-66. https://doi.org/10.1016/j.asr.2005.04.048

3. L. Taylor, H. Schmitt, W. Carrier, and M. Nakagawa, 1st AIAA Space EXP Conf, Orlando, Florida, 30 January- 01 February, 2005.

4. C. M. K. a. J. L. Edwards. Lunar dust transport and potential interactions with power system components; NASA/CR, 1991. https://doi.org/10.2514/6.2005-2510

5. E. Grün, M. Horanyi, and Z. Sternovsky, Planet Space Sci., 2011, 59, 1672-1680. https://doi.org/10.1016/j.pss.2011.04.005

6. M. J. E and C. D. R, Evidence for a high altitude distribution of lunar dust, Pergamon Press, Geochim. Cosmochim. Ac., 1974. http://ntrs.nasa.gov/search.jsp?R=19750055665

7. L. Xie, X. Zhang, L. Li, B. Zhou, Y. Zhang, Q. Yan, Y. Feng, D. Guo, and S. Yu, Geophys. Res. Lett., 2020, 47. https://doi.org/10.1029/2020gl089593

8. M. Horanyi, J. R. Szalay, S. Kempf, J. Schmidt, E. Grun, R. Srama, and Z. Sternovsky, Nature, 2015, 522, 324-326. https://doi.org/10.1038/nature14479

9. X. Wang, J. Schwan, H. W. Hsu, E. Grün, and M. Horányi, Geophys. Res. Lett., 2016, 43. 6103-6110. https://doi.org/10.1002/2016g1069491

10. O. R. Walton Adhesion of Lunar Dust; AIAA Glenn Research Center: NASA/CR, 2007

11. G. Barnes and I. Gentle, Interfacial Science: An Introduction. New York, Oxford University Press, 2011.

12. S. Brunauer, P. H. Emmett, and E. Teller, J. Am. Chem. Soc., 1938, 60, 309-319. https://doi.org/10.1021/ja01269a023

13. H. R. Moutinho, C. S. Jiang, B. To, C. Perkins, M. Muller, M. M. Al-Jassim, and L. Simpson, Sol. Energ. Mat. Sol. C, 2017, 172, 145-153. https://doi.org/10.1016/j.solmat.2017.07.026

14. A. Çolak, H. Wormeester, H. J. W. Zandvliet, and B. Poelsema, Appl. Surf. Sci., 2012, 258, 6938-6942. https://doi.org/10.1016/j.apsusc.2012.03.138

15. M. Farshchi-Tabrizia, M. Kappl, and H.-J. Butt, J. Adhes. Sci. Technol., 2008, 22, 181-203. https://doi.org/10.1163/156856108x306948

16. C. Pang and M. Bai, Appl. Surf. Sci., 2006, 253, 488-492. 10.1016/j.apsusc.2005.12.101

17. R. Alexander, Vacuum technology, Netherlands, Elsevier, 2012.

18. T. Eastman and D.-M. Zhu, Langmuir, 1996, 12, 2859-2862. https://doi.org/10.1021/la9504220 
19. A. Folch, M. S. Wrighton, and M. A. Schmidt, J. Microelectromech S, 1997, 6, 303-306. https://doi.org/10.1109/84.650126

20. H. Kimura, Astrophys J, 2016, 812, 67. https://doi.org/10.1088/0004-637X/821/1/67

21. J. L. Hutter and J. Bechhoefer, Rev. Sci. Instrum., 1993, 64, 1868-1873. https://doi.org/10.1063/1.1143970

22. S. M. Cook, K. M. Lang, K. M. Chynoweth, M. Wigton, R. W. Simmonds, and T. E. Schäffer, Nanotechnology, 2006, 17, 2135-2145. https://doi.org/10.1088/0957-4484/17/9/010

23. J. E. Sader, J. A. Sanelli, B. D. Adamson, J. P. Monty, X. Wei, S. A. Crawford, J. R. Friend, I. Marusic, P. Mulvaney, and E. J. Bieske, Rev. Sci. Instrum., 2012, 83, 103705. https://doi.org/10.1063/1.4757398
24. J. W. Marx and J. M. Sivertsen, J. Appl. Phys., 1953, 24, 81-87. https://doi.org/10.1063/1.1721138

25. V. M. Fokin and E. D. Zanotto, J. Non-Cryst. Solids, 2000, 265, 105-112. https://doi.org/ 10.1016/S0022-3093(99)00877-7

26. J. N. Israelachvili, in Intermolecular and Surface Forces. Netherlands, Elsevier, 2011.

27. Y. K. Shchipalov, Glass Ceram., 2000, 57, 11-12. https://doi.org/10.1023/A:1010900903019

28. M. Kimura, K. Nakamura, and K. Tsutsumi, J. Colloid Interface Sci., 2004, 279, 509-14. https://doi.org/10.1016/j.jcis.2004.06.093 